\title{
A Critical Analysis of Alternative Investment Opportunities Available, Risk and Return for High Networth Individuals
}

\author{
OMAR KHAMEES ABED
}

\author{
H.No, 10-1-18/14/2f, Shyam Nagar, Hyderabad-500028, Telangana, India Hyderabad, Telangana, India
}

\begin{abstract}
Portfolio Management has been an integral part for any investor. Each Investor, whether small or big is trying to maximize his/ her returns by making a diversified investment. To diversify the investment the risk and return trade off of each investment avenue has to be studied. Therefore the study of portfolio management and risk and return is very important of an investor. Now days there are professional investment advisors who study the risk and return portfolio of each investor and design the most well suited portfolio from him/ her. Banks also provide professional investment advice. This has opened up a huge market for the advisors and has become very competitive
\end{abstract}

Keywords: Organizational Effectiveness and Organizational Performance, Risk and Return Analysis, Portfolio Management.

\section{Introduction}

Risk management underscores the way that the survival of an association depends vigorously on its capacities to envision and plan for the change instead of simply sitting tight for the change and respond to it. The target of risk management is not to deny or counteract chance taking action, but rather to guarantee that the risks are deliberately brought with full information, reason and clear seeing so it can be measured and relieved. It additionally keeps an organization from affliction unsuitable misfortune bringing about a foundation to endure or physically harm its aggressive position. Elements of Risk management ought to really be bank particular directed by the size and nature of monetary record, intricacy of capacities, specialized/proficient labor and the status of MIS set up in that bank.

\section{Financial Management:}

Finance is an area of study which is namely concerned with two distinct areas i.e. financing and investing. We will be dealing with financial management relating to investment activities. This area of finance deals with finding out the best combination or portfolio of financial assets and thus focuses attention on the allocation of funds once they are acquired.

This area focuses attention whether an investor should put all his money in one financial asset or in a combination of different financial assets. It studies and addresses the ways in which individuals raise, allocate and use monetary resources over time, taking into account the risks entailed in their projects. It is the application of a set of techniques that individuals use to manage their financial affairs, particularly the differences between income and expenditure and the risks of their investments.

An entity whose income exceeds its expenditure can lend or invest the excess income. On the other hand, an entity whose income is less than its expenditure can raise capital by borrowing or selling equity claims, decreasing its expenses, or increasing its income. We will be focusing on those entities who have surplus income and are looking for avenues of investment. Therefore we will further go into portfolio management of individuals and risk and return analysis of each investment avenue.

\section{Portfolio Management:}

A portfolio is a mixture of investment avenues. An investor usually maintains a portfolio. The aim of managing a portfolio is to diversify and reduce risk and maximize return, which is also otherwise known as 'not putting all your eggs in a single basket'.

Portfolio Management is the processes, practices and specific activities to perform continuous and consistent evaluation, prioritization, budgeting, and finally selection of investments that provide the greatest value and contribution to the strategic interest of the investor. Through portfolio management, the investor can explicitly assess the tradeoffs among competing investment opportunities in terms of their benefit, costs, and risks.

The portfolio of each ING's HNI has been analyzed and on the basis of that various investment options open for these customers through the bank have been suggested. Each customer has the limit of risk he/she can undertake. This has also been looked into and under the limitations, which are the best options available have been advised. 


\section{HIGH NET WORTH INDIVIDUALS:}

A high net worth individual is a person with large personal financial holdings. Traditionally the term used was millionaire, but in recent years the term High Net Worth individual (HNI) has become the descriptor of choice.

A classification used by the financial services industry to denote an individual or a family with high net worth. Although there is no precise definition of how rich somebody must be to fit into this category, high net worth is generally quoted in terms of liquid assets over a certain figure. The exact amount differs by financial institution and region.

The categorization is relevant because high net worth individuals generally qualify for separately managed investment accounts instead of regular mutual funds. HNWI's are in high demand by private wealth managers. These individuals generally demand (and can justify) personalized services in investment management, estate planning, tax planning, and so on.

HNI's Investible assets refer to all fixed and current assets of the individual but exclude his primary dwelling. They include a simple savings or fixed deposit account with a financial institution, a securities account with a brokerage firm or an investment-linked policy with an insurance company. If the HNI has more than one account with the entity, the total net investible assets would mean the aggregate net invest able assets of the accounts.

\section{Services wanted by High Net Worth Individuals:}

HNI's are looking for professional advise on wealth management. This is termed as priority banking. Priority banking provides two major services to HNI's. First is wealth management and second is portfolio management.

Private Banking in a sense expands the definition of portfolio to wealth management. Wealth includes existing as well as fresh funds and apart from the traditional equity (both listed and private equity), debt and mutual funds, comprises art, real estate, jewellery etc.

The advantage banks have in providing this service is that there is a certain seamless ness in the entire operation, from making an investment decision to the proceeds, including charges being credited or debited to your account. Further, the essence of this business is security and confidentiality, which is what good banking essentially, is all about.

\section{RESEARCH}

The research design of this project is exploratory. Though each research study has its own specific purpose but the research design of this project on Hdfc Bank is exploratory in nature as the objective is the development of the hypothesis rather than their testing. The research designs methods of financial analysis. Through of comparative balance sheet in comparative statement, I am studying on balance sheet of Hdfc Bank. So taking comparative statement, I am going to analyzed of five years balance sheet of Hdfc Bank.

\section{Methodology}

The data used in this project is of secondary nature. The data is collected from secondary sources such as various websites, journals, newspapers, books, etc., the analysis used in this project has been done using selective technical tools. In Equity market, risk is analyzed and trading decisions are taken on basis of technical analysis. It is collection of share prices of selected companies for a period of five years.

In depth analysis of all the investment opportunities (instruments) that HDFC bank offers to its HNI clients. Also a sample study has been conducted on 20 HNI's of the bank to understand their individual risk appetite and investment preferences.

A comparison study of investment instruments offered by HDFC bank with that of other competitive banks has also been conducted. Based on these is an depth analysis was carried out to suggest/help the bank's investment advisors to cater to the needs of the HNI's in a better way thereby increasing the customers satisfaction and high HNI acquisition. 


\section{Data Analysis}

Table 0: Tabular Representation of various investment instruments (including SWOT analysis)

\begin{tabular}{|c|c|c|c|c|c|c|c|c|c|c|}
\hline Parameter & $\begin{array}{l}\text { Bank } \\
\text { Deposit }\end{array}$ & Equity & $\begin{array}{l}\text { Pref. } \\
\text { Share }\end{array}$ & MF & LIC & Govt Sec & $\begin{array}{l}\text { Corporat } \\
\text { e Bonds }\end{array}$ & Gold & Land & IPO \\
\hline ROR & $\begin{array}{l}\text { Between } \\
4-9 \%\end{array}$ & $5-20 \%$ & $5-20 \%$ & $10-25 \%$ & $4-5 \%$ & $5 \%$ & $5-6 \%$ & $\begin{array}{l}\text { No } \\
\text { fixed } \\
\text { return }\end{array}$ & $\begin{array}{l}\text { No fixed } \\
\text { Rate }\end{array}$ & $\begin{array}{l}\text { Depends } \\
\text { on } \\
\text { Demand \& } \\
\text { Supply }\end{array}$ \\
\hline Tenure & $\begin{array}{ll}\text { Long } & \\
\text { Term } & 10 \\
\text { vears }\end{array}$ & $\begin{array}{l}> \\
\text { years }\end{array}$ & $\begin{array}{l}> \\
\text { years }\end{array}$ & $1-5$ years & $\begin{array}{l}\text { Above } 10 \\
\text { years }\end{array}$ & $\begin{array}{l}\text { Lock in } \\
\text { period of } \\
6 \text { vears }\end{array}$ & $3-5$ years & $\begin{array}{l}\text { Above } \\
3 \text { years }\end{array}$ & $\begin{array}{l}5 \text { and } \\
\text { above }\end{array}$ & $\begin{array}{l}6 \text { mths }-3 \\
\text { years }\end{array}$ \\
\hline Security & $\begin{array}{l}\text { Secured } \\
\text { by Bank } \\
\text { regulated } \\
\text { by RBI }\end{array}$ & $\begin{array}{l}\text { No } \\
\text { security }\end{array}$ & $\begin{array}{l}\text { No } \\
\text { security }\end{array}$ & $\begin{array}{l}\text { No } \\
\text { security }\end{array}$ & $\begin{array}{l}\text { By issuing } \\
\text { organizati } \\
\text { on }\end{array}$ & $\begin{array}{l}\text { Secured } \\
\text { by } \\
\text { governme } \\
\text { nt }\end{array}$ & $\begin{array}{l}\text { Covered } \\
\text { under } \\
\text { rating } \\
\text { agencies }\end{array}$ & $\begin{array}{l}\text { Highly } \\
\text { secure } \\
\text { d }\end{array}$ & Secured & $\begin{array}{l}\text { No } \\
\text { security }\end{array}$ \\
\hline Liquidity & $\begin{array}{l}\text { Highly } \\
\text { liquid }\end{array}$ & Liquid & Liquid & $\begin{array}{l}\text { Provides } \\
\text { liquidity }\end{array}$ & $\begin{array}{l}\text { No } \\
\text { liquidity }\end{array}$ & $\begin{array}{l}\text { Provides } \\
\text { no } \\
\text { liquidity }\end{array}$ & $\begin{array}{l}\text { No } \\
\text { liquidity }\end{array}$ & $\begin{array}{l}\text { Highly } \\
\text { liquid }\end{array}$ & Not liquid & Liquid \\
\hline $\begin{array}{l}\text { Price } \\
\text { volatility }\end{array}$ & NIL & Maximum & Maximum & Maximum & NIL & NIL & NIL & $\begin{array}{l}\text { Moder } \\
\text { ate }\end{array}$ & Moderate & High \\
\hline Tax Benefit & $\begin{array}{l}\text { Tax } \\
\text { deducted } \\
\text { at source }\end{array}$ & $\begin{array}{l}\text { Long tem } \\
\text { capital } \\
\text { gains } \\
\text { benefit }\end{array}$ & $\begin{array}{l}\text { Long tem } \\
\text { capital } \\
\text { gains } \\
\text { benefit }\end{array}$ & $\begin{array}{l}\text { Long tem } \\
\text { capital } \\
\text { gains } \\
\text { benefit }\end{array}$ & $\begin{array}{l}\text { Benefit on } \\
\text { premium } \\
\text { amount } \\
\text { and } \\
\text { maturity } \\
\text { amount }\end{array}$ & $\begin{array}{l}\text { Interest } \\
\text { income } \\
\text { received } \\
\text { will be } \\
\text { taxable }\end{array}$ & Non & Non & $\begin{array}{l}\text { Only on } \\
\text { agricultur } \\
\text { al land }\end{array}$ & Nil \\
\hline $\begin{array}{l}\text { Risk profile } \\
\text { of an } \\
\text { investor }\end{array}$ & $\begin{array}{l}\text { Risk } \\
\text { averse }\end{array}$ & $\begin{array}{l}\text { Risk } \\
\text { seekers }\end{array}$ & $\begin{array}{l}\text { Risk } \\
\text { seekers }\end{array}$ & $\begin{array}{l}\text { Risk } \\
\text { seekers }\end{array}$ & $\begin{array}{l}\text { Low risk } \\
\text { seekers }\end{array}$ & $\begin{array}{l}\text { Risk } \\
\text { averse }\end{array}$ & $\begin{array}{l}\text { Moderate } \\
\text { risk }\end{array}$ & $\begin{array}{l}\text { Risk } \\
\text { averse }\end{array}$ & $\begin{array}{l}\text { Risk } \\
\text { averse }\end{array}$ & $\begin{array}{l}\text { Risk } \\
\text { averse }\end{array}$ \\
\hline Cash Flow & $\begin{array}{l}\text { Assured } \\
\text { cash flow }\end{array}$ & $\begin{array}{l}\text { No } \\
\text { assurity }\end{array}$ & $\begin{array}{l}\text { No } \\
\text { assurity }\end{array}$ & $\begin{array}{l}\text { No cash } \\
\text { flow }\end{array}$ & $\begin{array}{l}\text { Money } \\
\text { back } \\
\text { policy }\end{array}$ & $\begin{array}{l}\text { Maturity } \\
\text { amount } \\
\text { known }\end{array}$ & Non & Non & Non & NIL \\
\hline $\begin{array}{l}\text { Administra } \\
\text { tion }\end{array}$ & $\begin{array}{l}\text { Some } \\
\text { amount of } \\
\text { paper } \\
\text { work }\end{array}$ & Very less & Very less & $\begin{array}{l}\text { Paper } \\
\text { work is } \\
\text { there }\end{array}$ & $\begin{array}{l}\text { Cumberso } \\
\text { me paper } \\
\text { work }\end{array}$ & $\begin{array}{l}\text { Cumberso } \\
\text { me paper } \\
\text { work }\end{array}$ & Simple & Non & $\begin{array}{l}\text { Cumberso } \\
\text { me paper } \\
\text { work }\end{array}$ & $\begin{array}{ll}\text { A } & \text { lot } \\
\text { paper } & \\
\text { work } & \end{array}$ \\
\hline $\begin{array}{l}\text { Additional } \\
\text { features }\end{array}$ & $\begin{array}{l}\text { Pick- a- } \\
\text { date } \\
\text { feature }\end{array}$ & $\begin{array}{l}\text { Right to } \\
\text { vote in the } \\
\mathrm{CO}\end{array}$ & $\begin{array}{l}\text { Right to } \\
\text { vote in the } \\
\text { CO }\end{array}$ & Non & Life cover & Non & Non & $\begin{array}{l}\text { Multip } \\
\text { le } \\
\text { usage }\end{array}$ & $\begin{array}{l}\text { Multiple } \\
\text { usage }\end{array}$ & Non \\
\hline Collateral & $\begin{array}{l}\text { Highly } \\
\text { accepted }\end{array}$ & $\begin{array}{l}\text { Only } \\
\text { available } \\
\text { on loans } \\
\text { against } \\
\text { shares }\end{array}$ & $\begin{array}{l}\text { Only } \\
\text { available } \\
\text { on loans } \\
\text { against } \\
\text { shares }\end{array}$ & $\begin{array}{l}\text { Not } \\
\text { accepted }\end{array}$ & Accepted & Accepted & Non & $\begin{array}{l}\text { Accept } \\
\text { ed }\end{array}$ & $\begin{array}{l}\text { Mainly } \\
\text { provided }\end{array}$ & No \\
\hline $\begin{array}{l}\text { Professiona } \\
\text { l } \\
\text { Manageme } \\
\text { nt }\end{array}$ & $\begin{array}{l}\text { Profession } \\
\text { ally } \\
\text { Managed }\end{array}$ & $\begin{array}{l}\text { Profession } \\
\text { ally } \\
\text { Managed }\end{array}$ & $\begin{array}{l}\text { Profession } \\
\text { ally } \\
\text { Managed }\end{array}$ & $\begin{array}{l}\text { Profession } \\
\text { ally } \\
\text { Managed }\end{array}$ & $\begin{array}{l}\text { Profession } \\
\text { ally } \\
\text { Managed }\end{array}$ & $\begin{array}{l}\text { Managed } \\
\text { by } \\
\text { Goverm } \\
\text { ent }\end{array}$ & $\begin{array}{l}\text { Profession } \\
\text { ally } \\
\text { Managed }\end{array}$ & $\begin{array}{l}\text { Not } \\
\text { manag } \\
\text { ed }\end{array}$ & $\begin{array}{l}\text { Not } \\
\text { managed }\end{array}$ & $\begin{array}{l}\text { Profession } \\
\text { ally } \\
\text { Managed }\end{array}$ \\
\hline
\end{tabular}

\section{Analysis of Each Investment Avenue}

In the investing world, the dictionary definition of risk is the chance that an investment's actual return will be different than expected. Technically, this is measured in statistics by standard deviation. Risk means you have the possibility of losing some, or even all, of our original investment. Given below is the table, which states the kind of risk, return, tenure and other benefits of different investment avenues. This helps us further suggest the kind of investment an investor can make based on the personal profile.

Table 1: kind of risk, return, tenure and other benefits of different investment avenues

\begin{tabular}{|l|l|l|l|l|}
\hline Type of investments & Risk & Returns & Tenure & Other benefits \\
\hline Equity & High & High & Long & Low \\
\hline Gold & Low & Moderate & Long & High \\
\hline Preference Shares & High & High & Long & Low \\
\hline Corporate Bonds & Low & Low & Moderate & Low \\
\hline Mutual Funds & High & High & Short & Low \\
\hline Government Securities & Low & Low & Long & High \\
\hline Land & Low & High & Long & High \\
\hline Bank Deposits & Low & Moderate & Moderate & High \\
\hline IPO's & High & High & Short & Low \\
\hline Insurance Policy & Low & Low & Long & High \\
\hline
\end{tabular}

\section{AWARENESS OF REGULATIONS}

1. What is your assessment of your readiness for the new Basel proposals with respect to capital requirements? 
Table 2: Readiness for the new Basel proposal

\begin{tabular}{|l|l|l|l|}
\hline & CREDT RISK & MARKET RISK & OPERATIONAL RISK \\
\hline FULLY PREPARED & 8 & 9 & 9 \\
\hline PARTIALLY PREPARED & 2 & 1 & 1 \\
\hline NOT YET PREPARED & & & \\
\hline
\end{tabular}

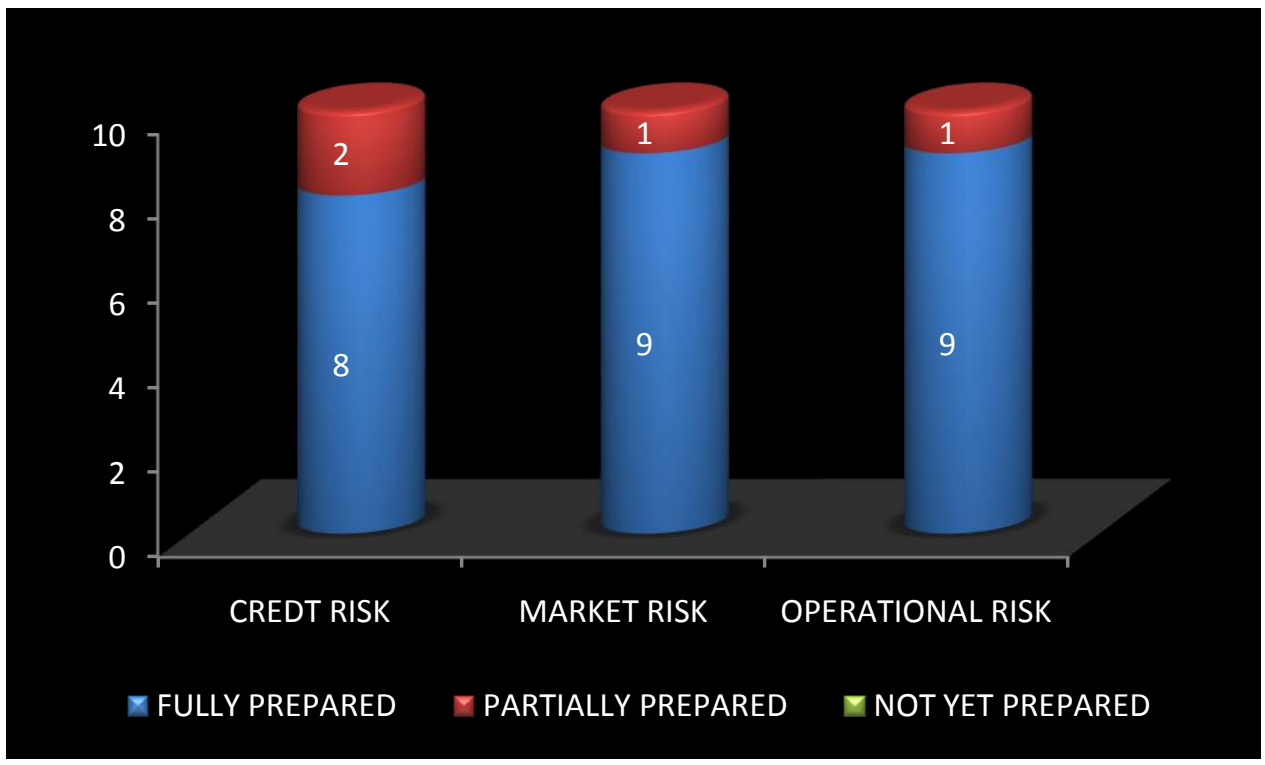

Figure 1 Readiness for the new Basel proposal

2. Have you done a gap analysis between current risk management practice and new capital requirements?

Table 3: Gap analysis

\begin{tabular}{|l|l|l|l|}
\hline & CREDT RISK & MARKET RISK & OPERATIONAL RISK \\
\hline YES & 9 & 5 & 9 \\
\hline NO & 1 & 5 & 1 \\
\hline
\end{tabular}

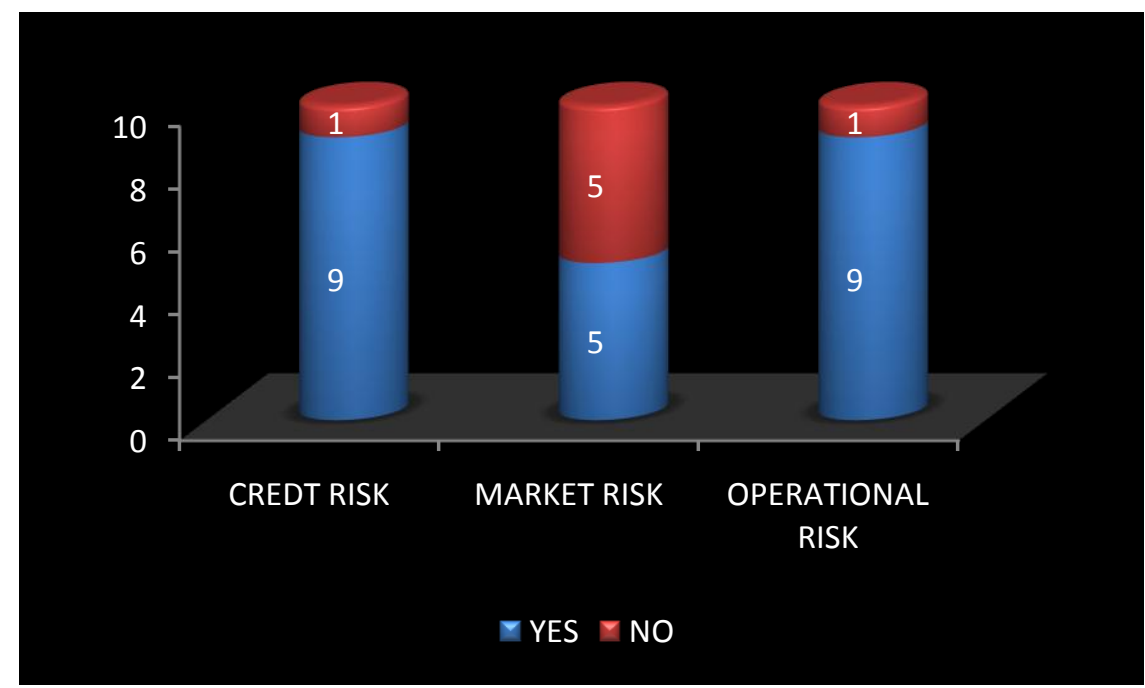

Figure 2 Gap analysis

3. What degree of priority do you address to the new Basel regulatory framework?

Table 4: Priority to new Basel regulatory framework

\begin{tabular}{|l|l|l|l|}
\hline & CREDT RISK & MARKET RISK & OPERATIONAL RISK \\
\hline VERY IMPORTANT & 9 & 9 & 10 \\
\hline IMPORTANT & 1 & 1 & \\
\hline NOT IMPORTANT & & & \\
\hline
\end{tabular}




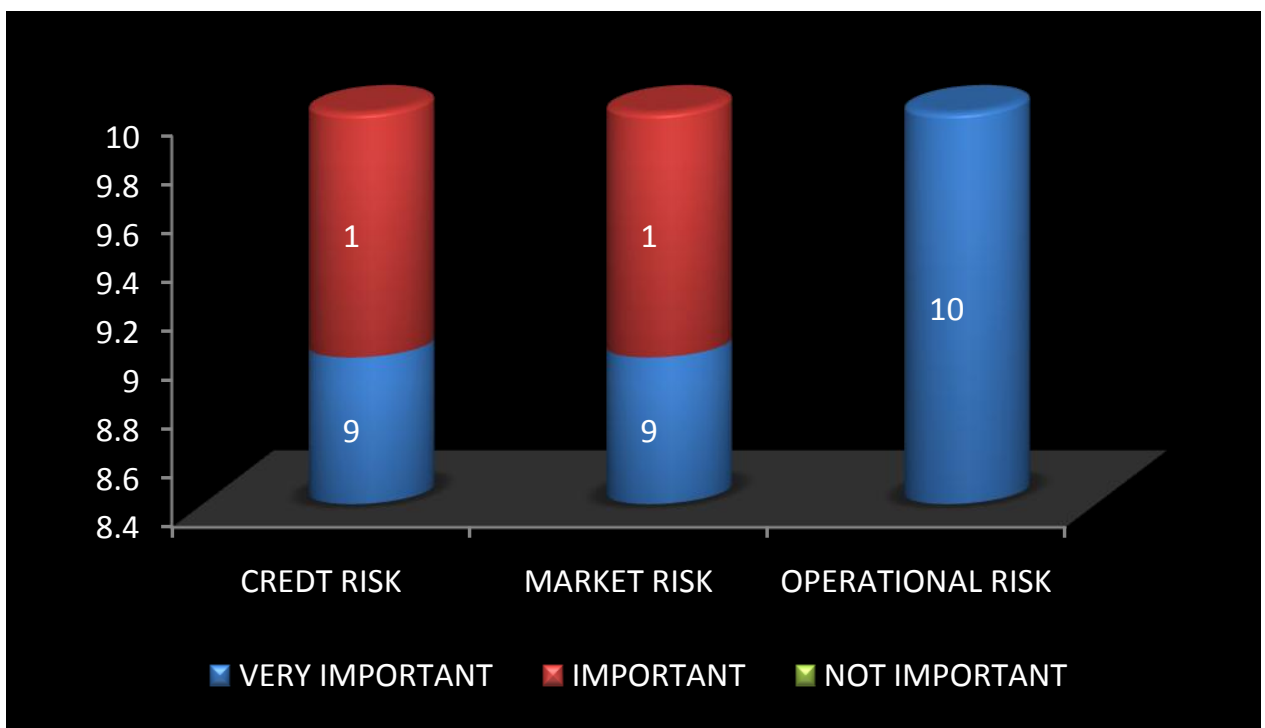

Figure 3 Priority to new Basel regulatory framework

4. How do you view Basel II regulation: as an opportunity to enhance the risk management process, or as a regulatory constraint?

Table 5: View of Basel II regulation
\begin{tabular}{|l|l|l|l|}
\hline & CREDT RISK & MARKET RISK & OPERATIONAL RISK \\
\hline OPPORTUNITY & 10 & 8 & 10 \\
\hline CONSTRAINT & & 2 & \\
\hline
\end{tabular}

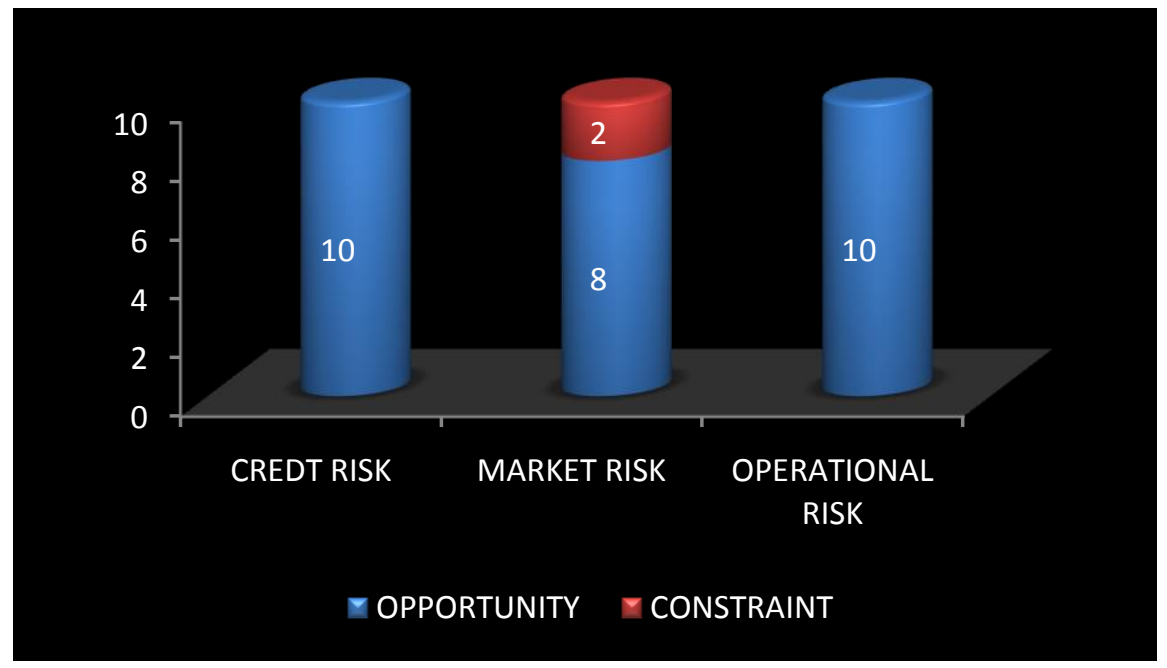

Figure 4: View of Basel II regulation

\section{ORGANISTIONAL STRUCTURE}

1. Do you have an assigned Credit risk, Market risk and Operational risk manager in your bank?

Table 6: Assignment of risk manager

\begin{tabular}{|l|l|l|l|}
\hline & CREDT RISK & MARKET RISK & OPERATIONAL RISK \\
\hline YES & 10 & 9 & 9 \\
\hline NO & & 1 & 1 \\
\hline
\end{tabular}




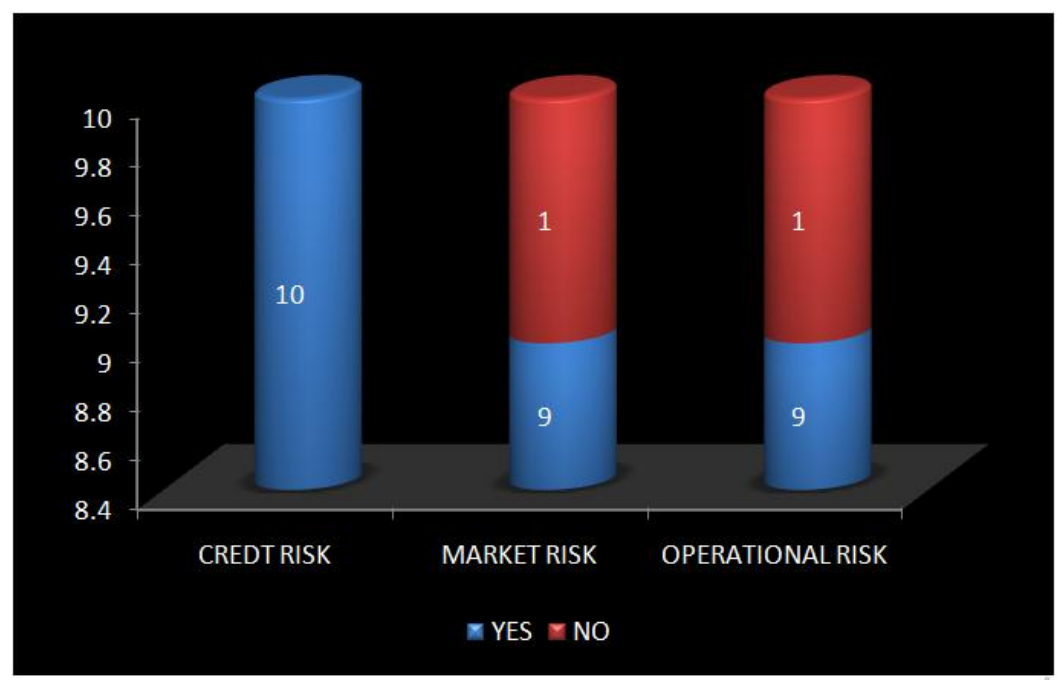

Figure 5: Assignment of risk manager

2. To whom does the Risk manager report?

Table 7: Whom does risk manager report

\begin{tabular}{|l|l|l|l|}
\hline & CREDT RISK & MARKET RISK & OPERATIONAL RISK \\
\hline CHIEF EXECUTIVE OFFICER & 4 & 6 & 6 \\
\hline CHIEF FINANCIAL OFFICER & & & \\
\hline ASSETS AND LIABLITY MANAGER & 2 & 1 & 1 \\
\hline CREDIT RISK OFFICER & 4 & 2 & 2 \\
\hline OTHER SPECIFY & & & \\
\hline
\end{tabular}

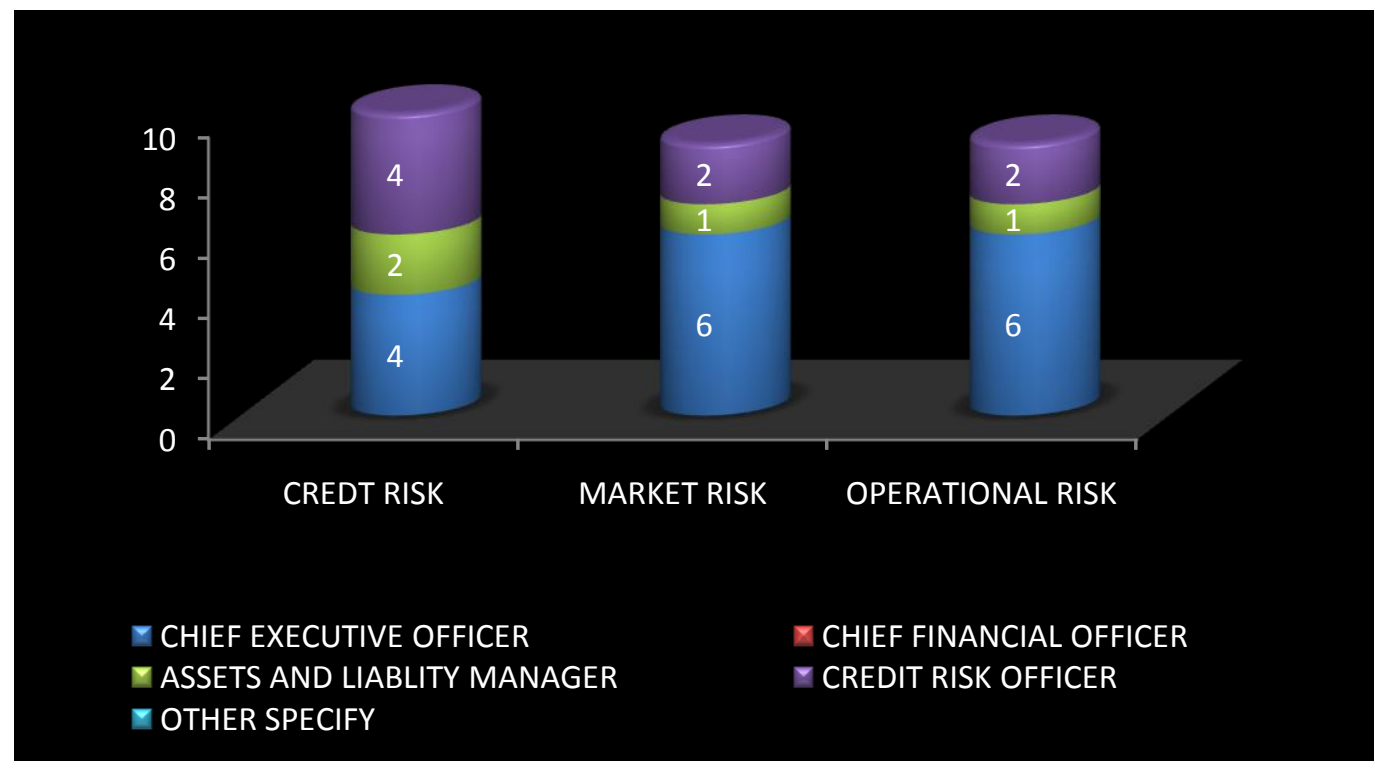

Figure 6: Whom does risk manager report?

3. What is the assigned manager's time dedicated to this activity?

Table 8: Time dedication

\begin{tabular}{|l|l|l|l|}
\hline & CREDT RISK & MARKET RISK & OPERATIONAL RISK \\
\hline $0-20 \%$ & 2 & 4 & 2 \\
\hline $20-50 \%$ & 2 & 1 & 2 \\
\hline$>50 \%$ & 6 & 5 & 6 \\
\hline
\end{tabular}




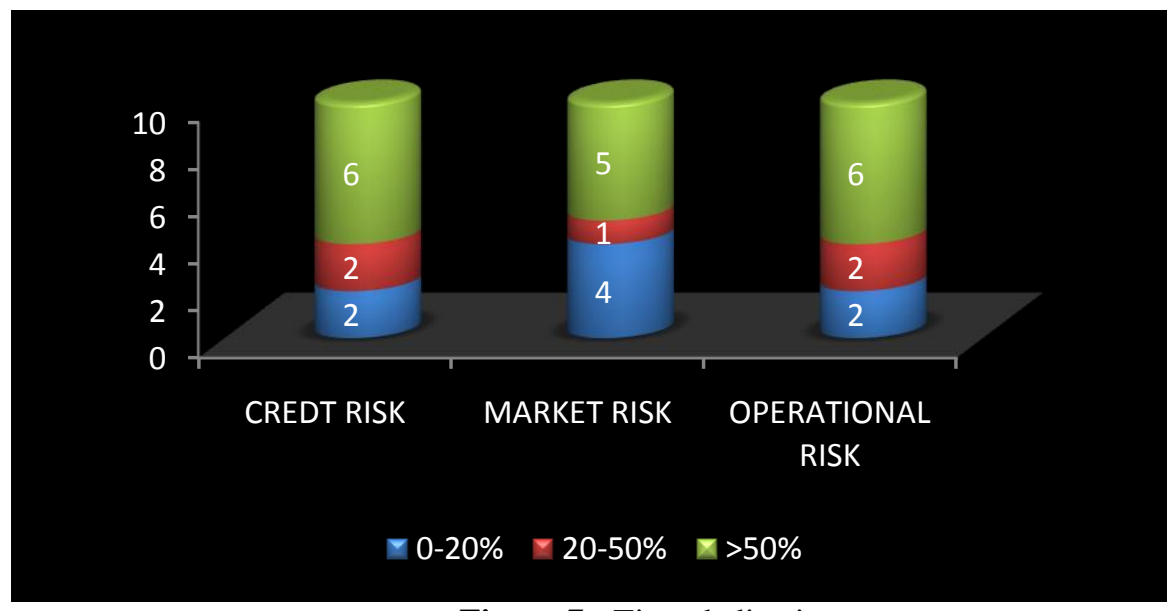

Figure 7: Time dedication

4. How many people work in these departments?

Table 9: Number of people work

\begin{tabular}{|c|l|l|l|}
\hline & CREDT RISK & MARKET RISK & OPERATIONAL RISK \\
\hline $1-3$ & 2 & 4 & 1 \\
\hline $3-5$ & 6 & 4 & 5 \\
\hline $5-10$ & 1 & 1 & 1 \\
\hline$>10$ & 1 & 1 & 3 \\
\hline
\end{tabular}

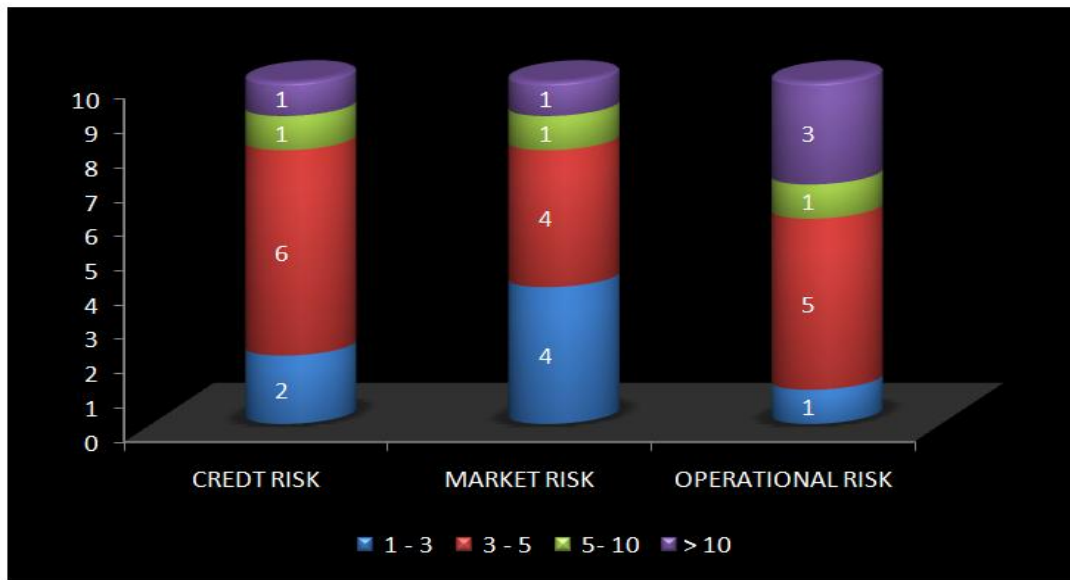

Figure 8: Number of people work

5. Do you have a Risk Committee?

Table 10: Risk Committee

\begin{tabular}{|l|l|l|l|}
\hline & CREDT RISK & MARKET RISK & OPERATIONAL RISK \\
\hline YES & 6 & 5 & 6 \\
\hline NO & 4 & 5 & 4 \\
\hline
\end{tabular}

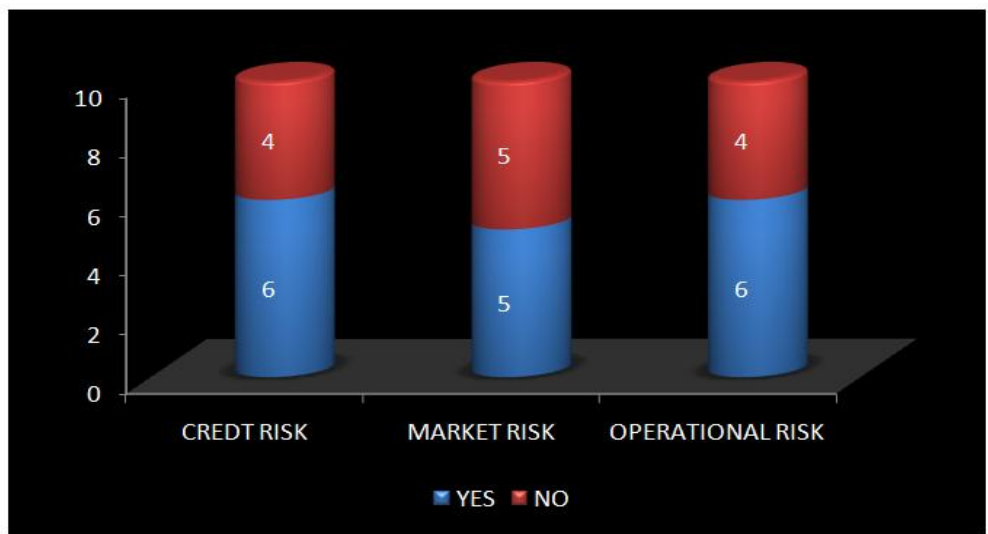

Figure 9: Risk Committee 


\section{OBSERVATIONS}

- Almost all of the participating banks have a risk management department.

- Most of the industry's risk managers' report to the Chief Executive Officer, Asset and liability manager and Chief Risk Officer accounting for the balance in equal proportions.

- Slightly more attention is paid to credit and operational risk than to Market risk, as $40 \%$ of the banks operating do not have risk committee.

\section{INTERPRETATION}

- Despite the relatively small size of banks, they are generally well aware of the risk management function, and for this purpose, risk managers spend over half their time performing these functions.

\section{REPORTING ABILITY}

1. Are you producing reporting for

Table 11: Reports produced for

\begin{tabular}{|l|l|l|l|}
\hline & CREDT RISK & MARKET RISK & OPERATIONAL RISK \\
\hline REGULATORY PURPOSE & 3 & 4 & 4 \\
\hline MONITORING & 7 & 8 & 8 \\
\hline DECISION MAKING PURPOSE & 7 & 4 & 4 \\
\hline
\end{tabular}

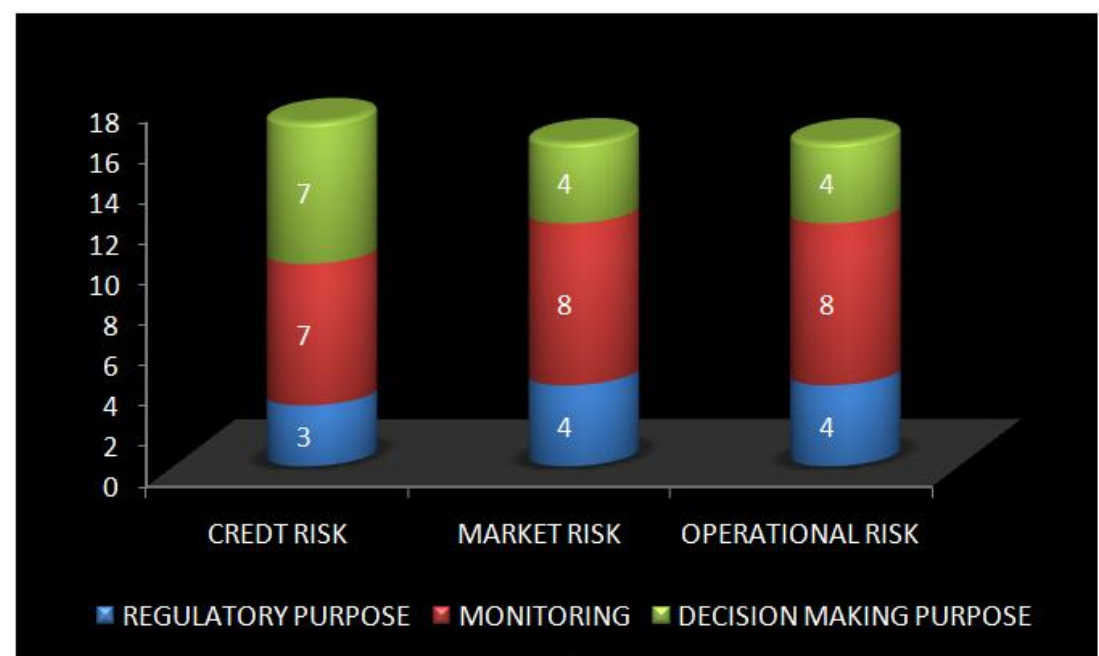

Figure 10: Reports produced for

2. Does external reporting drive your internal reporting?

Table 12: External reporting drive internal reporting

\begin{tabular}{|l|l|l|l|}
\hline & CREDT RISK & MARKET RISK & OPERATIONAL RISK \\
\hline VERY SIGNIFICANTLY & 4 & 5 & 4 \\
\hline SIGNIFICANTLY & 5 & 4 & 5 \\
\hline NOT AT ALL SIGNIFICANTLY & 1 & 1 & 1 \\
\hline
\end{tabular}

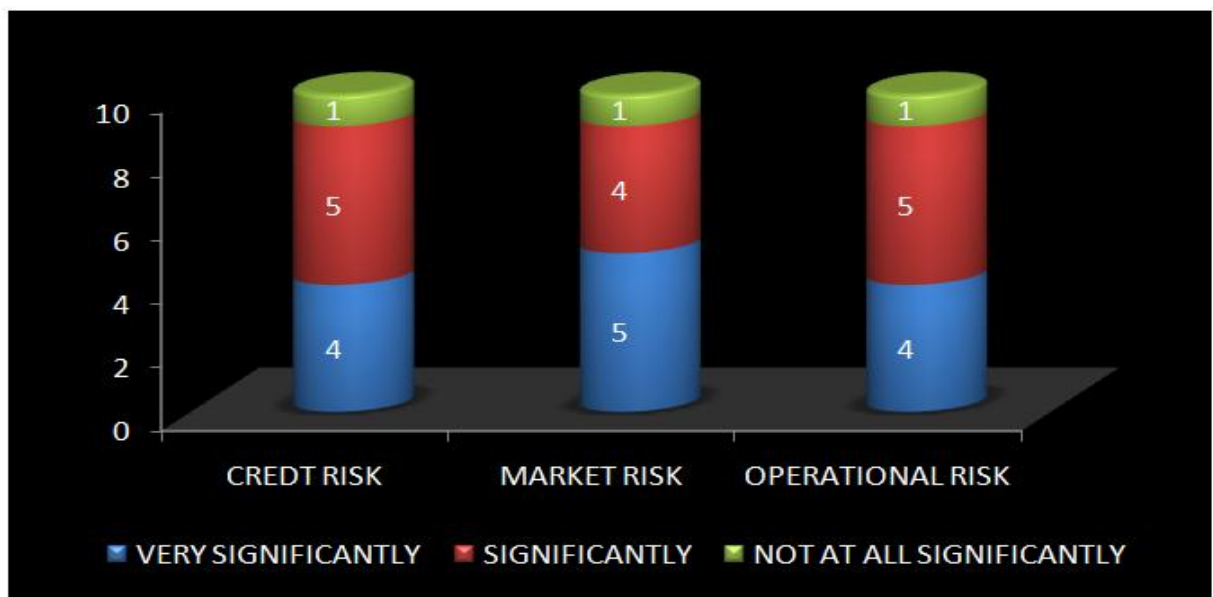

Figure 11: External reporting drive internal reporting 
3. Does external reporting affect your decision making process?

Table 13: External reporting affect decision making process

\begin{tabular}{|l|l|l|l|}
\hline & CREDT RISK & MARKET RISK & OPERATIONAL RISK \\
\hline VERY SIGNIFICANTLY & 3 & 3 & 3 \\
\hline SIGNIFICANTLY & 6 & 5 & 6 \\
\hline NOT AT ALL SIGNIFICANTLY & 1 & 2 & 1 \\
\hline
\end{tabular}

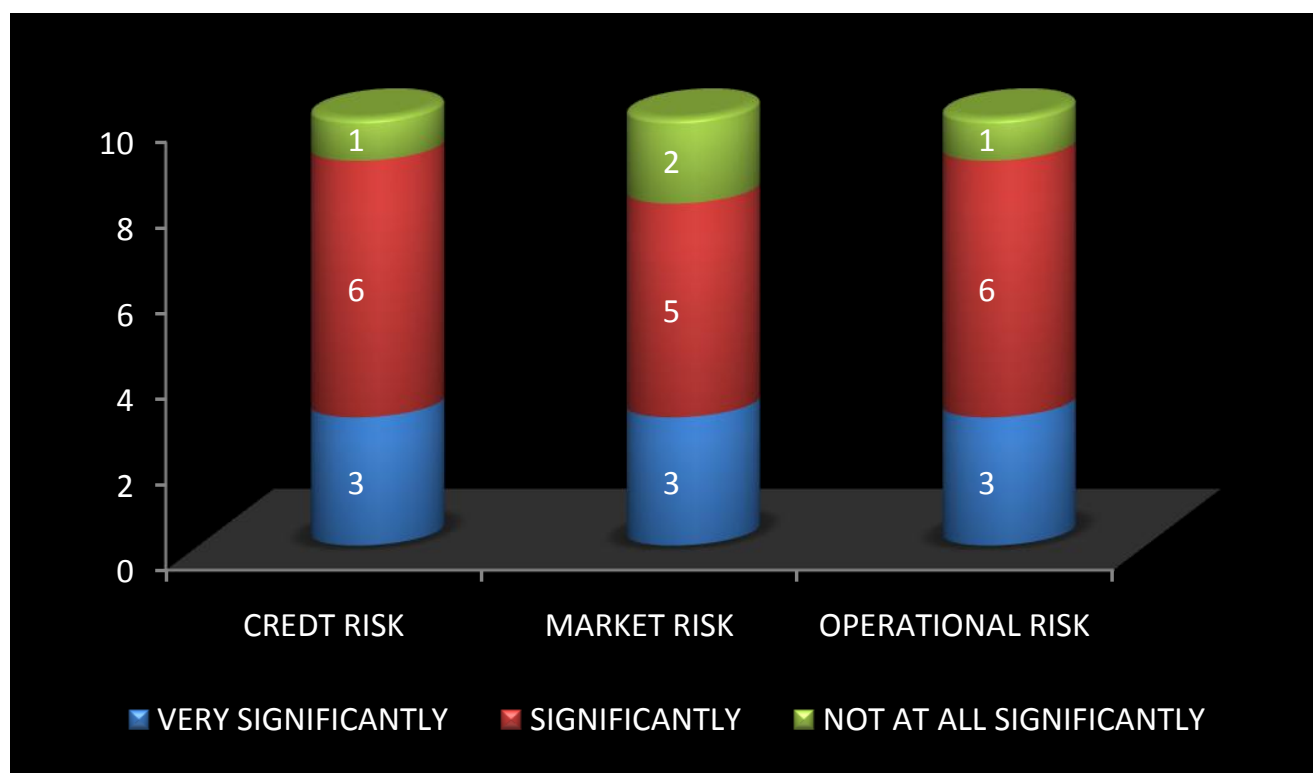

Figure 12: External reporting affect decision making process

4. How frequent is your internal reporting?

Table 14: Frequency of internal reporting

\begin{tabular}{|l|l|l|l|}
\hline & CREDT RISK & MARKET RISK & OPERATIONAL RISK \\
\hline Daily & 1 & & 1 \\
\hline Weekly & & 1 & 1 \\
\hline Monthly & 8 & 8 & 7 \\
\hline Annually & 1 & 1 & 1 \\
\hline
\end{tabular}

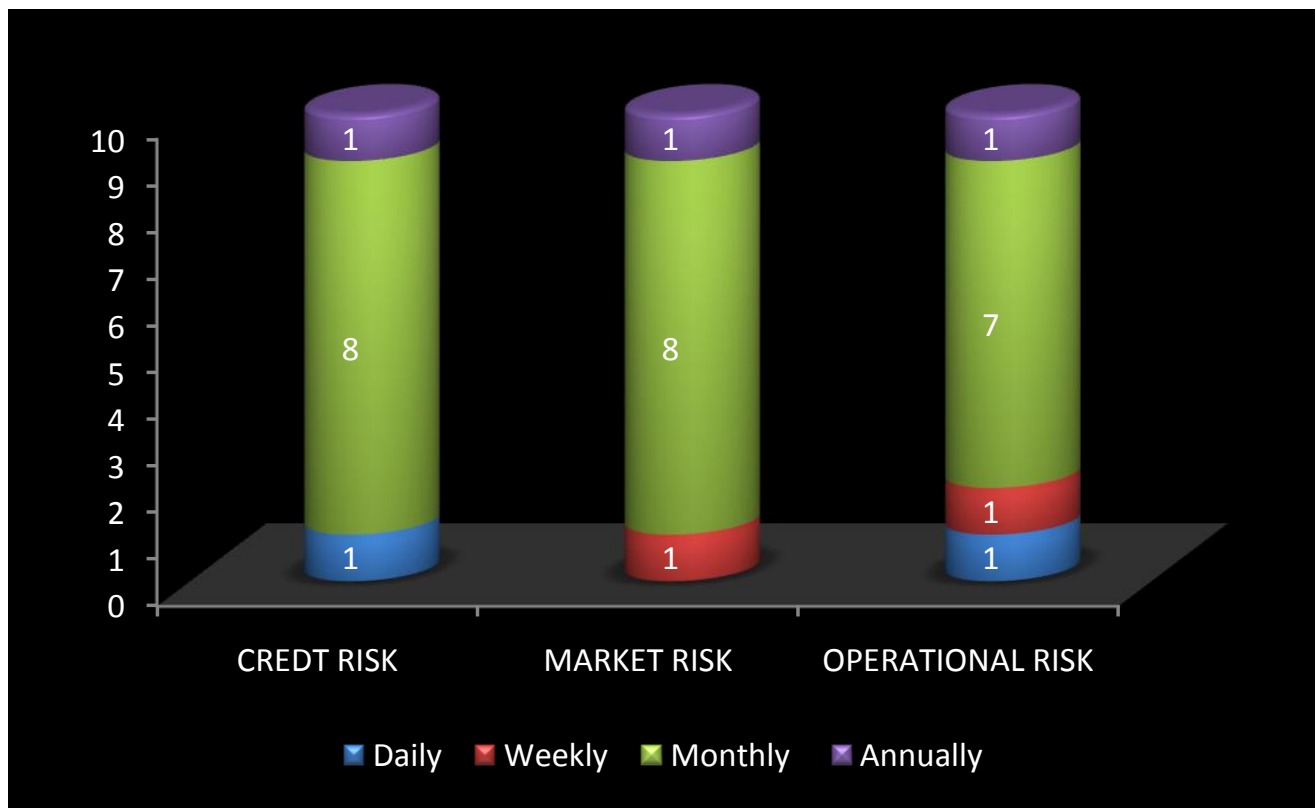

Figure 13: Frequency of internal reporting

5. Will you produce specific internal reporting for Credit, Market and Operational Risk? 
Table 15: Production of specific internal reporting

\begin{tabular}{|l|l|l|l|}
\hline & CREDT RISK & MARKET RISK & OPERATIONAL RISK \\
\hline YES & 10 & 10 & 10 \\
\hline NO & & & \\
\hline
\end{tabular}

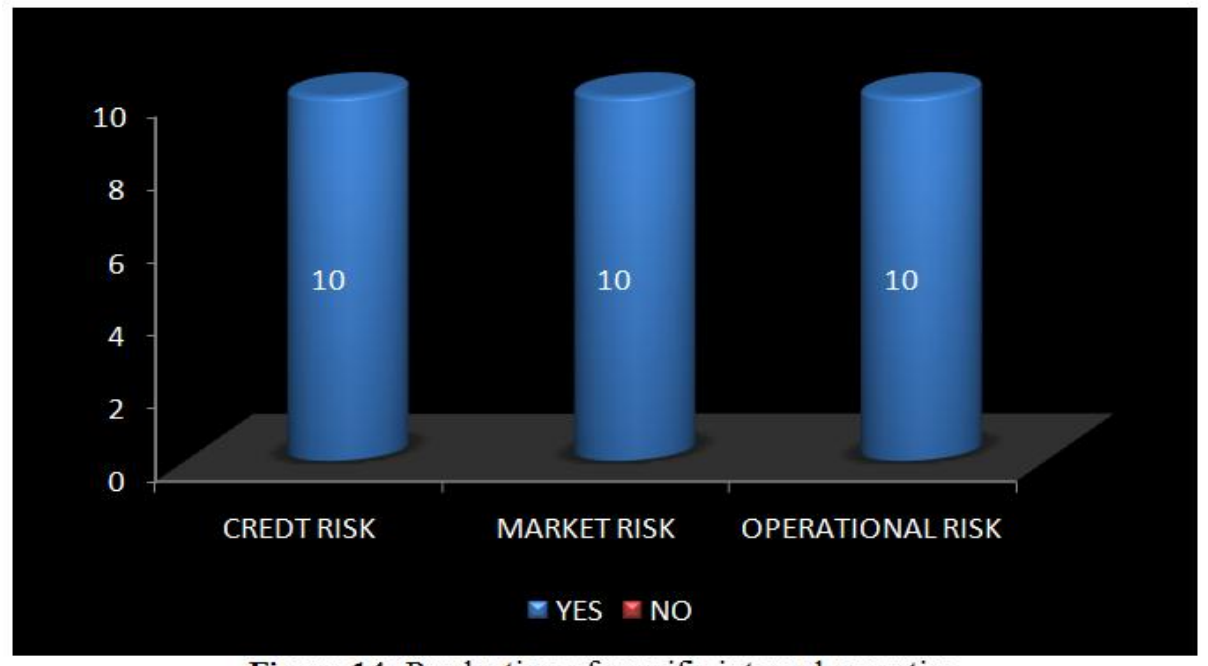

Figure 14: Production of specific internal reporting

\section{Conclusion}

The project report analyzes the portfolio of ING's HNI customers. A sample study of 10 HNI's was conducted and on the basis of which the recommendations were made. The products and services of the bank are compared to other banks also. It has been noticed that HNI's provide a huge opportunity to the RM's of the bank for giving professional investment advice. Now a days the customers are well aware of the surrounding market and don't really take any type of advice. They are looking for investment advice, which are supported by strong conviction. The market of professional advisors are growing at such a huge rate that if the client is not happy with the services of the RM he/she can easily look for a better advisors. Therefore the RM's along with personal advice have to also provide quality advice. An enthusiastic relationship manager should show computation of the risk of HNI's by using statistical computations like Standard Deviation, which shows how much on an average, the return has moved away from an average number. Basic tents one should understand before taking a risk argument to a client. Risk, popularly measured by Standard Deviation, tells us about the range of return that can probably occur. Its interpretation is appropriate for an asset class. For example if we say that equity is more risky than PPF, we will substantiate that by saying that the probability that the investor will get a return different than $8 \%$ is low in a PPF. However, in equity while he may look at a $20 \%$ coverage. It can swing between $4 \%$ and $46 \%$, indicating the presence of risk. The risk to the investor is always distilled in terms of his goals, investments and the target rate that his investments must achieve to meet the goal. Therefore, the investor will not care for standard deviation in itself, but about the risk that his investment will fall short. If one is planning for the child's education or retirement, such shortfalls are too tough to make good, on a later date. So, to the investor, risk is only about down side deviation from a target rate. How does one put the two together? The choice of asset classes and percentage allocation will have to be driven by standard deviation and its understanding. But the subsequent monitoring and review of the portfolio will have to be in terms of the investor's goals, and the possibility that such allocation will fall short. Movement from one stock to another is unlikely to alter the risk profile of the portfolio in any significant manner. But adjusting the proportion that is invested in equity is likely to impact both the risk and return of the portfolio. Relationship managers need to train their eyes to watch if the investor's goals are best served by the current allocation, or should a change be made, given downside risks. Learning investment concepts is all fine, but to apply them to an investor's situation, one has to view the portfolio from the investor's angle rather. My project does not go into the details of calculating the risk and returns of each investment avenue, as it would have been very technical and cumbersome. The sample study of 10 does not really show the true findings, as there could be other clients with different opinions about the investment instruments than the ones stated. 


\section{Reference}

[1] Bodie, Kane, Marcus and Mohanty, "Investments", 8th Edition., Tata McGraw Hill Private Limited, New Delhi, 2009

[2] Jorion, Philippe, "Value at Risk : The new benchmark for managing financial risk", $3{ }^{\text {rd }}$ Edition, McGraw Hill, 2006

[3] Ackermann, C., R. McNally and D. Ravenscraft, "The performance of hedge funds:Risk, return and incentives", Journal of Finance", Vol No. 54(3), June 1999

[4] Sortino, Frank. A and Lee N. Price, "Performance measurement in a downside risk framework", "Journal of Investing" Vol. 3(3), Fall 1994.

[5] Stutzer, M, “A portfolio performance index”, "Financial Analysts Journal”, Vol. 56(3), May-June 2000.

[6] "Handbook of Statistics on the Indian Economy" by Reserve Bank of India - 2008.

[7] Agarwal, V., \& Naik, N. Y. (2000). On taking the "alternative" route: the risks, rewards, and performance persistence of hedge funds. The Journal of Alternative Investments, 2(4), 6-23.

[8] Schneeweis, T., Karavas, V. N., \& Georgiev, G. (2002). Alternative investments in the institutional portfolio. Alternative Investment Management Association.

[9] Lakonishok, J., Shleifer, A., \& Vishny, R. W. (1994). Contrarian investment, extrapolation, and risk. The journal of finance, 49(5), 1541-1578.

IOSR Journal of Business and Management (IOSR-JBM) is UGC approved Journal with S1. No. 4481, Journal no. 46879

Omar. K. "A Critical Analysis of Alternative Investment Opportunities Available, Risk and Return for High Networth Individuals." IOSR Journal of Business and Management (IOSR-JBM) 19.7 (2017): $102-112$. 